\title{
Overcoming the Electroluminescence Efficiency Limitations of Perovskite Light-Emitting Diodes
}

Authors: Himchan $\mathrm{Cho}^{1 \dagger}{ }^{1 \dagger}$, Su-Hun Jeong ${ }^{1 \dagger}$, Min-Ho Park ${ }^{1 \dagger}$, Young-Hoon Kim ${ }^{1}$, Christoph Wolf $^{1}$, Chang-Lyoul Lee ${ }^{2}$, Jin Hyuck Heo ${ }^{3}$, Aditya Sadhanala ${ }^{4}$, NoSoung Myoung ${ }^{2}$, Seunghyup Yoo ${ }^{5}$, Sang Hyuk $\mathrm{Im}^{3}$, Richard H. Friend ${ }^{4}$, Tae-Woo Lee ${ }^{1,6} *$

\section{Affiliation:}

${ }^{1}$ Department of Materials Science and Engineering, Pohang University of Science and Technology (POSTECH), 77 Cheongam-Ro, Pohang, Gyungbuk 790-784, Republic of Korea.

${ }^{2}$ Advanced Photonics Research Institute (APRI), Gwangju Institute of Science \& Technology (GIST), 1 Oryong-dong, Buk-gu, Gwangju, 500-712, Republic of Korea.

${ }^{3}$ Department of Chemical Engineering, College of Engineering, Kyung Hee University, 1 Seochon-dong, Giheung-gu, Youngin-si, Gyeonggi-do 446-701, Republic of Korea.

${ }^{4}$ Cavendish Laboratory, University of Cambridge, JJ Thomson Avenue, Cambridge CB3 OHE, UK.

${ }^{5}$ Department of Electrical Engineering, Korea Advanced Institute of Science and Technology (KAIST), 373-1 Guseong-dong, Daejeon 305-701.

${ }^{6}$ Department of Chemical Engineering, Division of Advanced Materials Science, School of Environmental Science and Engineering, Pohang University of Science and Technology (POSTECH), 77 Cheongam-Ro, Nam-Gu, Pohang, Gyungbuk 790-784, Republic of Korea.

*Corresponding author. E-mail: twlee@ postech.ac.kr, taewlees@gmail.com

$\dagger:$ These authors contributed equally to this work.

Abstract: Organic-inorganic hybrid perovskites are emerging low-cost emitters with very high color purity, but their low luminescent efficiency is a critical drawback. We boosted the current efficiency (CE) of perovskite LEDs with a simple bilayer structure to 42.9 candela per ampere, similar to CE of phosphorescent OLEDs, with two modifications. We prevented the formation of metallic $\mathrm{Pb}$ atoms that cause significant exciton quenching through small increase in methylammonium bromide (MABr) molar proportion, and we spatially confined the exciton in uniform $\mathrm{MAPbBr}_{3}$ nanograins (average diameter $=99.7$ nanometers) formed by a nanocrystal pinning process and concomitant reduction of exciton diffusion length to 67 nanometers. These changes caused substantial increases in steady-state photoluminescence intensity and lifetime of $\mathrm{MAPbBr}_{3}$ nanograin layers.

One Sentence Summary: Ultrahigh-efficiency organic/inorganic hybrid perovskite lightemitting diodes of $42.9 \mathrm{~cd} \mathrm{~A}^{-1}$ was achieved using stoichiometric tuning and nanograin engineering.

Main Text: Organic-inorganic hybrid perovskites (OIPs) have recently established as important class of materials in photovoltaic devices with rapid progress in increasing their power conversion efficiency (1-5). OIPs are being emerged also as promising light emitters because they can provide very high color purity (full width at half maximum $\sim 20 \mathrm{~nm}$ ) irrespective of the crystal size, unlike conventional inorganic quantum dots, because their intrinsic crystal structure is similar to a multiple quantum well $(6,7)$. Also, OIPs have low material cost and simply- 
tunable band gap with a reasonable ionization energy (IE) comparable to common hole injection materials (7-11). Thus, OIPs are attractive materials as alternative emitters that can overcome the disadvantages of organic light-emitting diodes (OLEDs) (e.g., complex synthesis, high cost, and poor color purity) and inorganic quantum dot LEDs (e.g., complex synthesis, high cost and high IE).

Bright electroluminescence $(\mathrm{EL})\left(>100 \mathrm{~cd} \mathrm{~m}^{-2}\right)$ at room temperature from perovskite light-emitting diodes (PeLEDs) with methylammonium lead halides ( $\mathrm{MAPbX}_{3}$, where $\mathrm{X}$ is $\mathrm{I}, \mathrm{Br}$ or $\mathrm{Cl}$ ) emission layer was demonstrated recently $(6,7,12-18)$. As an emission layer, $\mathrm{MAPbBr}_{3}$ has higher air stability $(7,19)$ and exciton binding energy $(76$ or $150 \mathrm{meV})$ than does $\mathrm{MAPbI}_{3}$ (30 or $50 \mathrm{meV})(20,21)$. However, PeLEDs have much lower current efficiency (CE) at room temperature than do OLEDs or quantum dot LEDs. Existing methods have not overcome the substantial luminescence quenching in $\mathrm{MAPbX}_{3}$ caused by facile thermal ionization of excitons generated in the OIP layer, which has a low exciton binding energy. Spin-coating of $\mathrm{MAPbBr}_{3}$ solution creates a rough, non-uniform surface with many cuboids with large grain size (22), which leads to a substantial leakage current and large exciton diffusion length $L_{D}$ that reduces CE in PeLEDs. To improve the CE of PeLEDs, the OIP grain size must be decreased, and OIP films should be flat and uniform. Smaller grains can spatially limit $L_{D}$ of excitons or charge carriers and reduce the possibility of exciton dissociation into carriers. This fabrication goal differs from that of the OIP layers in solar cells, which should be dense films with large grain size to achieve good exciton diffusion and dissociation. Thus, processes designed to achieve uniform OIP film morphology with large grain size in solar cells such as solvent dripping $(23,24)$ are not applicable to PeLEDs, which require small $L_{D}$.

Here, we report a systematic approach for achieving highly bright and efficient green PeLEDs with $\mathrm{CE}=42.9 \mathrm{~cd} \mathrm{~A}^{-1}$ and external quantum efficiency $(\mathrm{EQE})=8.53 \%$ even in a simplified bilayer structure. These high efficiencies represent $>20,000$-fold increase compared with that of the control devices and are higher than the best EQE of a previous report regarding visible PeLEDs using OIP films by factors of > 10.6 (Table S1, Fig. S1) (15). The high efficiency PeLEDs were constructed based on effective management of exciton quenching by a modified $\mathrm{MAPbBr}_{3}$ emission layer that was achieved using (i) fine and controllable stoichiometry modification and (ii) optimized nanograin engineering by nanocrystal pinning (NCP) (Fig. S2). Furthermore, we demonstrated a flexible PeLED using a self-organized conducting polymer (SOCP) anode and the first large-area PeLED ( $2 \mathrm{~cm}$ by $2 \mathrm{~cm}$ pixel).

A fundamental problem that must be solved to achieve high CE in PeLEDs is minimizing the presence of metallic $\mathrm{Pb}$ atoms in $\mathrm{MAPbBr}_{3}$ that limits the efficiency of PeLEDs. Metallic $\mathrm{Pb}$ atoms can emerge in $\mathrm{MAPbBr}_{3}$ even if $\mathrm{MABr}$ and $\mathrm{PbBr}_{2}$ are mixed with 1:1 (mol:mol) ratios because of the decomposition of MABr or incomplete reaction between $\operatorname{MABr}$ and $\operatorname{PbBr}_{2}(7,19)$. Excess $\mathrm{Pb}$ atoms degrade luminescence by increasing the nonradiative decay rate and decreasing the radiative decay rate (25). Prevention of the formation of metallic $\mathrm{Pb}$ atoms was achieved by finely increasing the molar proportion of $\mathrm{MABr}$ by 2 to $7 \%$ in $\mathrm{MAPbBr}_{3}$ solution (Fig. S2A). Use of excess MABr suppressed exciton quenching and reduced hole-injection barrier from SOCP layers (Table S2) to $\mathrm{MAPbBr}_{3}$ layers with decreased IE and greatly increased steady-state photoluminescence (PL) intensity and $\mathrm{PL}$ lifetime of $\mathrm{MAPbBr}_{3}$ films. We propose that radiative decay of the $\mathrm{PL}$ process in $\mathrm{MAPbBr}_{3}$ nanograins originates from shallow-trap-assisted radiative recombination at grain boundaries and radiative recombination inside the grains. Second, the $\mathrm{CE}$ in PeLEDs can be increased by decreasing $\mathrm{MAPbBr}_{3}$ grain sizes, which improves uniformity and 
coverage of $\mathrm{MAPbBr}_{3}$ nanograin layers and radiative recombination by confining the excitons in the nanograins (leading to small $L_{D}$ ). An optimized NCP process (Fig. S3) helped change in the morphology of $\mathrm{MAPbBr}_{3}$ layers from scattered micrometer-sized cuboids to well-packed nanograins with uniform coverage, which greatly reduced leakage current and increased CE.

We fabricated $\mathrm{MAPbBr}_{3}$ films by spin-coating using stoichiometrically-modified perovskite solutions on prepared glass/SOCPs or silicon wafer/SOCPs substrates later used in devices (Fig. 1, A and B), and then characterized the films' morphologies and optoelectronic properties. The solutions had different molar ratios of $\mathrm{MABr}$ to $\mathrm{PbBr}_{2}\left(\mathrm{MABr}_{\mathrm{PbBr}}=1.05: 1\right.$, 1:1, or 1:1.05). To achieve uniform surface coverage and reduced grain size, NCP was used instead of normal spin-coating (Fig. S3). This process washed out the "good" solvents [dimethylformamide or dimethyl sulfoxide (DMSO)], and causes pinning of NCs by inducing fast crystallization. Chloroform was chosen as the solvent for NCP because a highly volatile nonpolar solvent is suitable to reduce the size and increase the uniformity of $\mathrm{MAPbBr}_{3}$ grains by reducing solvent evaporation time. In addition, to further reduce grain size, we devised additivebased NCP (A-NCP) which uses an organic small molecule, 2,2',2"-(1,3,5-benzinetriyl)-tris(1phenyl-1-H-benzimidazole) (TPBI), as an additive to chloroform, whereas pure chloroform is used in solvent-based NCP (S-NCP).

Use of NCP affected film morphology (Fig. 2). Without NCP, micrometer-sized $\mathrm{MAPbBr}_{3}$ cuboids were scattered on the SOCP layer (Fig. 2A). They were only interconnected with a few other cuboids, and so a large amount of space remained uncovered. This high surface roughness and the formation of pinholes in OIP films result in formation of bad interface with the electron transport layer and electrical shunt paths, and thus severely limit CE in PeLEDs. In contrast, when NCP was used, perfect surface coverage was obtained, and the $\mathrm{MAPbBr}_{3}$ crystal morphology changed to a well-packed assembly of tiny grains ranging from 100 to $250 \mathrm{~nm}$ (Fig. 2, B to E, Fig. S4). MAPbBr 3 grain size was little affected by the stoichiometric modification of $\mathrm{MAPbBr}_{3}$ solutions (Fig. 2, B to D, Fig. S4, A to C). Furthermore, $\mathrm{MAPbBr}_{3}$ grain size was further reduced to $50-150 \mathrm{~nm}$ (average $=99.7 \mathrm{~nm}$ ) by A-NCP (Fig. 2E, Fig. S4D). This reduction can be attributed to hindrance of crystal growth by TPBI molecules during crystal pinning. The thickness of $\mathrm{MAPbBr}_{3}$ layer was $400 \mathrm{~nm}$ (Fig. 1B).

The crystal structures of $\mathrm{MAPbBr}_{3}$ films were analyzed by measuring $\mathrm{x}$-ray diffraction (XRD) patterns (Fig. 2F, Fig. S5, Table S3). The XRD patterns of MAPbBr films $_{3}(1: 1)$ exhibit peaks at $15.02^{\circ}, 21.3^{\circ}, 30.28^{\circ}, 33.92^{\circ}, 37.24^{\circ}, 43.28^{\circ}$ and $46.00^{\circ}$ that can be assigned to $(100)$, (110), (200), (210), (211), (220) and (300) planes respectively, by using Bragg's law to convert the peak positions to interplanar spacings (Fig. 2F). The lattice parameter is in accordance with a previous report (19), and demonstrates that $\mathrm{MAPbBr}_{3}$ films had a stable cubic $P m \overline{3} m$ phase. Using the Scherrer equation, the crystallite size was calculated to be $23.5 \pm 2.0 \mathrm{~nm}$, and the variation with stoichiometric change was not large (Table S3). Because the crystallite sizes were much smaller than the apparent grain sizes (Fig. 2, A to E), we conclude that all grains consisted of many crystallites. The stoichiometric changes had very little effect on the peak positions (Fig. S5A). Furthermore, A-NCP did not change the peak positions when compared to S-NCP (Fig. S5); this stability in positions indicates that the stoichiometric changes of $\mathrm{MAPbBr}_{3}$ solution and the use of TPBI additive did not affect the crystal structure of $\mathrm{MAPbBr}_{3}$ films.

To study chemical changes in the $\mathrm{MAPbBr}_{3}$ layers fabricated using perovskite solutions with different stoichiometries, $\mathrm{X}$-ray photoelectron spectroscopy (XPS) was conducted. The survey spectra showed strong peaks of $\mathrm{Br}(\sim 68 \mathrm{eV}), \mathrm{Pb}(\sim 138$ and $143 \mathrm{eV}), \mathrm{C}(\sim 285 \mathrm{eV})$ and $\mathrm{N}$ 
$(\sim 413 \mathrm{eV})$; these results agree with values in previous reports (Fig. S6A) (7, 26-28). Systematic deconvolution of $\mathrm{Pb} 4 \mathrm{f}, \mathrm{Br} 3 \mathrm{~d}$ and N1s spectra into summations of Gaussian-Lorentzian curves revealed the nature of chemical bonds in $\mathrm{MAPbBr}_{3}$ (Fig. S6, B to D, Fig. S7). The gradual increase of $\mathrm{MABr}$ molar proportion in the films was confirmed by observing the gradual increase in N1s peak intensities as MABr:PbBr 2 increased from 1:1.05 to 1.05:1 (Fig. S7, C and D) and the gradual decrease in $\mathrm{Br}: \mathrm{Pb}$ atomic ratio (Supplementary Text F). In the Pb4f spectra (Fig. S6, B to F), large peaks were observed at $\sim 138.8$ and $\sim 143.6 \mathrm{eV}$ (caused by the spin orbit split) that correspond to $\mathrm{Pb}_{4 / 2}$ and $\mathrm{Pb}_{7 / 2}$ levels, respectively (26-28). Each of these peaks was associated with a smaller peak that was shifted to $1.8-\mathrm{eV}$ lower binding energy; these small peaks can be assigned to metallic $\mathrm{Pb}$ (26-28). The height of peaks that represent metallic $\mathrm{Pb}$ decreased as MABr:PbBr 2 increased from 1:1.05 to 1:1 (Fig. S6, E and F); this peak was absent in the film with $\mathrm{MABr}: \mathrm{PbBr}_{2}=1.05: 1$ (Fig. S6F). This trend indicates that the presence of metallic $\mathrm{Pb}$ atoms on the films was successfully prevented by fine stoichiometry control. In contrast, the high peak intensity of the metallic $\mathrm{Pb}$ peak in the films with $\mathrm{MABr}: \mathrm{PbBr}_{2}=1: 1$ and 1:1.05 suggests that numerous metallic $\mathrm{Pb}$ atoms were formed on the film surfaces.

We measured the work functions (WFs) and IEs of the $\mathrm{MAPbBr}_{3}$ films using ultraviolet photoelectron spectroscopy (UPS) (Fig. S8). The WFs were obtained by subtracting the energies at secondary cut-offs of the UPS spectra from the UV radiation energy of $21.2 \mathrm{eV}$ when a Fermi level of $0 \mathrm{eV}$ was the common reference for all energies. The IEs were determined by adding the WF (Fig. S8A) to the energy offset between WFs and IEs of MAPbBr 3 (Fig. S8B) (29). The IE gradually decreased with increasing $\mathrm{MABr}$ molar proportion from $6.01 \mathrm{eV}$ in the film with MABr:PbBr $2=1: 1.05$ to $5.86 \mathrm{eV}$ in the film with $\mathrm{MABr}: \mathrm{PbBr}_{2}=1.1: 1$ (Fig. 1C, Table S4). The gradual decrease in IEs with decreasing $\mathrm{PbBr}_{2}$ molar proportion can be understood based on the IE being greater in $\mathrm{PbBr}_{2}$ than in $\mathrm{MAPbBr}_{3}$ (30). In PeLEDs, this decrease can help alleviate hole-injection barriers from SOCP layers to $\mathrm{MAPbBr}_{3}$ layers (Fig. 1C).

The luminescent properties of the $\mathrm{MAPbBr}_{3}$ films were investigated by steady-state PL measurement (Fig. 3A). The measurement was conducted using a spectrofluorometer with excitation from monochromatic light with wavelength of $405 \mathrm{~nm}$ (xenon lamp). The $\mathrm{MAPbBr}_{3}$ films fabricated from $\mathrm{MABr}: \mathrm{PbBr}_{2}=1.05: 1$ had a $~ 5.8$ times increase in PL intensity (Fig. 3A) compared with 1:1 films, and had much higher PL quantum efficiency (PLQE; $36 \%$ vs $3 \%$ ). In addition, the reduction of grain size with A-NCP vs S-NCP increased the PL intensity by $\sim 2.8$ times. The PL intensity of the films with $\mathrm{MABr}: \mathrm{PbBr}_{2}=1: 1.05$ was greater than in those with $\mathrm{MABr}: \mathrm{PbBr}_{2}=1: 1$, although the $\mathrm{PbBr}_{2}$ molar proportion had increased in the former. We suspect that this departure from the expected trend is due to $\mathrm{PbBr}_{2}$-induced surface passivation of the film; this process reduces nonradiative recombination at the trap sites (31).

To understand the kinetics of excitons and free carriers in $\mathrm{MAPbBr}_{3}$ films and how the presence of metallic $\mathrm{Pb}$ atoms affects the PL lifetime, time-correlated single-photon counting (TCSPC) measurements were conducted (Fig. 3B). The PL decay curves were fitted using a biexponential decay model, in which the PL lifetime is considered as the summation of fast and slow decay components that give short lifetime $\tau_{1}$ and long lifetime $\tau_{2}$, respectively. To investigate the quality of quenching sites, we prepared the layers $\left(\mathrm{MABr}: \mathrm{PbBr}_{2}=1.05: 1\right)$ with and without sealing with a 50-nm-thick poly(methyl methacrylate) (PMMA) layer. The fraction $f_{2}$ of $\tau_{2}$ decreased from $91 \%$ to $77 \%$ in the film without sealing (Table S5). Oxygen and moisture can diffuse quickly into grain boundaries when the top PMMA layer is not used; oxygen or moisture at grain boundaries provides quenching sites. The fast decay is related to 
trap-assisted recombination at grain boundaries, whereas the slow decay is related to radiative recombination inside the grains (Fig. S9) (32).

This proposition was supported by analyzing the change in $\tau$ and $f$ of $\mathrm{MAPbBr}_{3}$ films with varying stoichiometric ratio. As $\mathrm{MABr}: \mathrm{PbBr}_{2}$ increased from 1:1 to 1.05:1, the average lifetime $\tau_{\text {avg }}$ gradually increased from 12.1 to $51.0 \mathrm{~ns}$ (Table S5). The short $\tau_{\text {avg }}(12.1 \mathrm{~ns})$ in the film with $\mathrm{MABr}: \mathrm{PbBr}_{2}=1: 1$ originated from the significant reduction of $\tau_{2}$. This implies that uncoordinated metallic $\mathrm{Pb}$ atoms at grain boundaries inhibit radiative recombination and cause strong nonradiative recombination (Fig. S9). The $\mathrm{MAPbBr}_{3}$ films fabricated using $\mathrm{PbBr}_{2}$-rich perovskite solution $\left(\mathrm{MABr}: \mathrm{PbBr}_{2}=1: 1.05\right)$ had a longer lifetime than films with $\mathrm{MABr}: \mathrm{PbBr}_{2}=$ 1:1, possibly through $\mathrm{PbBr}_{2}$-induced surface passivation (31). We calculated the average $L_{D}$ using a model similar to that in previous reports (Fig. S10) (33). The films $\left(\mathrm{MABr}_{\mathrm{PbBr}} \mathrm{Pb}_{2}\right.$ 1.05:1) underneath a PMMA layer exhibited a much smaller $L_{D}(67 \mathrm{~nm})$ than those previously reported $(>1 \mu \mathrm{m})(33,34)$. We attribute this reduction in $L_{D}$ to the reduced grain sizes in which excitons are spatially stronger confined, thereby reducing dissociation and enhancing radiative recombination; this compensates the plausible adverse effect of larger grain boundary area (6).

The PeLED fabricated without using NCP showed poor luminous characteristics (maximum $\mathrm{CE}=2.03 \times 10^{-3} \mathrm{~cd} \mathrm{~A}^{-1}$ ) mainly due to high leakage current (Fig. S11). In contrast, maximum CE was significantly increased $\left(0.183 \mathrm{~cd} \mathrm{~A}^{-1}\right)$ when a full-coverage uniform $\mathrm{MAPbBr}_{3}$ nanograin layer with decreased grain size was achieved using S-NCP (Fig. 4, A and B, Table 1). The maximum CE was boosted up to $21.4 \mathrm{~cd} \mathrm{~A}^{-1}$ in the PeLEDs fabricated with perovskite solutions with excess $\operatorname{MABr}(1.07: 1,1.05: 1,1.03: 1$ and 1.02:1) (Fig. 4A, Table 1). As $\mathrm{MABr}: \mathrm{PbBr}_{2}$ increased from 1:1 to $1.05: 1$, the maximum CE varied from 0.183 to $21.4 \mathrm{~cd} \mathrm{~A}^{-1}$. Without stoichiometric modifications of $\mathrm{MAPbBr}_{3}$ to avoid metallic $\mathrm{Pb}$ atoms (molar ratios 1:1 and 1:1.05), the achieved maximum CEs was only 0.183 and $4.87 \times 10^{-2} \mathrm{~cd} \mathrm{~A}^{-1}$, respectively.

We further increased CE of PeLEDs by using A-NCP. The PeLEDs based on A-NCP had a maximum CE of $42.9 \mathrm{~cd} \mathrm{~A}^{-1}$ (Fig. 4, C and D, Table 1), which represents an EQE of $8.53 \%$ when the angular emission profile is considered (Fig. S12). The EL spectra of PeLEDs were very narrow; full width at half maximum was $\sim 20 \mathrm{~nm}$ for all spectra; this high color purity of OIP emitters show great potential when used in displays (Fig. 4E). A pixel of the PeLED of based on $\mathrm{MABr}: \mathrm{PbBr}_{2}=1.05: 1$ exhibited strong green light emission (Fig. S13A). Furthermore, the proposed processes and materials used therein are compatible with flexible and large-area devices; a high-brightness flexible PeLED (Fig. 4, F and G) and a large-area ( $2 \mathrm{~cm}$ by $2 \mathrm{~cm}$ pixel) PeLED (Fig. S13B) were fabricated. Our study reduces the technical gap between PeLEDs and OLEDs or quantum dot LEDs, and is a big leap towards the development of efficient nextgeneration emitters with high color purity and low fabrication cost based on perovskites.

\section{References and Notes:}

1. W. S. Yang, J. H. Noh, N. J. Jeon, Y. C. Kim, S. Ryu, J. Seo, S. I. Seok, High-performance photovoltaic perovskite layers fabricated through intramolecular exchange. Science 348, 1234-1237 (2015).

2. M. Liu, M. B. Johnston, H. J. Snaith, Efficient planar heterojunction perovskite solar cells by vapour deposition. Nature 501, 395-398 (2013).

3. N. J. Jeon, J. H. Noh, W. S. Yang, Y. C. Kim, S. Ryu, J. Seo, S. I. Seok, Compositional 
engineering of perovskite materials for high-performance solar cells. Nature 517, 476-480 (2015).

4. J.-H. Im, I.-H. Jang, N. Pellet, M. Grätzel, N.-G. Park, Growth of $\mathrm{CH}_{3} \mathrm{NH}_{3} \mathrm{PbI}_{3}$ cuboids with controlled size for high-efficiency perovskite solar cells. Nat. Nanotechnol. 9, 927-932 (2014).

5. J.-Y. Jeng, K.-C. Chen, T.-Y. Chiang, P.-Y. Lin, T.-D. Tsai, Y.-C. Chang, T.-F. Guo, P. Chen, T.-C. Wen, Y.-J. Hsu, Nickel oxide electrode interlayer in $\mathrm{CH}_{3} \mathrm{NH}_{3} \mathrm{PbI}_{3}$ perovskite/PCBM planar-heterojunction hybrid solar cells. Adv. Mater. 26, 4107-4113 (2014).

6. Z.-K. Tan, R. S. Moghaddam, M. L. Lai, P. Docampo, R. Higler, F. Deschler, M. Price, A. Sadhanala, L. M. Pazos, D. Credgington, F. Hanusch, T. Bein, H. J. Snaith, R. H. Friend, Bright light-emitting diodes based on organometal halide perovskite. Nat. Nanotechnol. 9 , 687-692 (2014).

7. Y.-H. Kim, H. Cho, J. H. Heo, T.-S. Kim, N. Myoung, C.-L. Lee, S. H. Im, T.-W. Lee, Multicolored organic/inorganic hybrid perovskite light-emitting diodes. Adv. Mater. 27, 7, 12481254 (2015).

8. D. B. Mitzi, Synthesis, crystal structure, and optical and thermal properties of $\left(\mathrm{C}_{4} \mathrm{H}_{9} \mathrm{NH}_{3}\right)_{2} \mathrm{MI}_{4}(\mathrm{M}=\mathrm{Ge}, \mathrm{Sn}, \mathrm{Pb})$. Chem. Mater. 8, 791-800 (1996).

9. M. R. Filip, G. E. Eperon, H. J. Snaith, F. Giustino, Steric engineering of metal-halide perovskites with tunable optical band gaps. Nat. Commun. 5, 5757 (2014).

10. T. M. Koh, K. Fu, Y. Fang, S. Chen, T. C. Sum, N. Mathews, S. G. Mhaisalkar, P. P. Boix, T. Baikie, Formamidinium-containing metal-halide: an alternative material for near-IR absorption perovskite solar cells. J. Phys. Chem. C. 118, 16458-16462 (2014).

11. G. E. Eperon, S. D. Stranks, C. Menelaou, M. B. Johnston, L. M. Herz, H. J. Snaith, Formamidinium lead trihalide: a broadly tunable perovskite for efficient planar heterojunction solar cells. Energy Environ. Sci. 7, 982-988 (2014).

12. R. L. Z. Hoye, M. R. Chua, K. P. Musselman, G. Li, M.-L. Lai, Z.-K. Tan, N. C. Greenham, J. L. MacManus-Driscoll, R. H. Friend, D. Credgington, Enhanced performance in fluorenefree organometal halide perovskite light-emitting diodes using tunable, low electron affinity oxide electron injectors. Adv. Mater. 27, 1414-1419 (2015).

13. N. K. Kumawat, A. Dey, K. L. Narasimhan, D. Kabra, Near infrared to visible electroluminescent diodes based on organometallic halide perovskites: structural and optical investigation. ACS Photonics 2, 349-354 (2015).

14. G. Li, Z.-K. Tan, D. Di, M. L. Lai, L. Jiang, J. H.-W. Lim, R. H. Friend, N. C. Greenham, Efficient light-emitting diodes based on nanocrystalline perovskite in a dielectric polymer matrix. Nano Lett. 15, 2640-2644 (2015).

15. J. Wang, N. Wang, Y. Jin, J. Si, Z.-K. Tan, H. Du, L. Cheng, X. Dai, S. Bai, H. He, Z. Ye, M. L. Lai, R. H. Friend, W. Huang, Interfacial control toward efficient and low-voltage perovskite light-emitting diodes. Adv. Mater. 27, 2311-2316 (2015).

16. A. Sadhanala, A. Kumar, S. Pathak, A. Rao, U. Steiner, N. C. Greenham, H. J. Snaith, R. H. Friend, Electroluminescence from organometallic lead halide perovskite-conjugated polymer diodes. Adv. Electron. Mater. 1, 1500008 (2015). 
17. J. C. Yu, D. B. Kim, G. Baek, B. R. Lee, E. D. Jung, S. Lee, J. H. Chu, D.-K. Lee, K. J. Choi, S. Cho, M. H. Song, High-performance planar perovskite optoelectronic devices: a morphological and interfacial control by polar solvent treatment. Adv. Mater. 27, 3492-3500 (2015).

18. N. K. Kumawat, A. Dey, A. Kumar, S. P. Gopinathan, K. L. Narasimhan, D. Kabra, Band gap tuning of $\mathrm{CH}_{3} \mathrm{NH}_{3} \mathrm{~Pb}\left(\mathrm{Br}_{1-\mathrm{x}} \mathrm{Cl}_{\mathrm{x}}\right)_{3}$ hybrid perovskite for blue electroluminescence. ACS Appl. Mater. Interfaces, 7, 13119-13124 (2015).

19. J. H. Noh, S. H. Im, J. H. Heo, T. N. Mandal, S. I. Seok, Chemical management for colorful, efficient, and stable inorganic-organic hybrid nanostructured solar cells. Nano Lett. 13, 17641769 (2013).

20. I. B. Koutselas, L. Ducasse, G. C. Papavassiliou, Electronic properties of three- and lowdimensional semiconducting materials with $\mathrm{Pb}$ halide and $\mathrm{Sn}$ halide units. J. Phys.: Condens. Matter 8, 1217-1227 (1996).

21. K. Tanaka, T. Takahashi, T. Ban, T. Kondo, K. Uchida, N. Miura, Comparative study on the excitons in lead-halide-based perovskite-type crystals $\mathrm{CH}_{3} \mathrm{NH}_{3} \mathrm{PbBr}_{3} \mathrm{CH}_{3} \mathrm{NH}_{3} \mathrm{PbI}_{3}$. Solid State Commun. 127, 619-623 (2003).

22. J. H. Heo, D. H. Song, S. H. Im, Planar $\mathrm{CH}_{3} \mathrm{NH}_{3} \mathrm{PbBr}_{3}$ hybrid solar cells with $10.4 \%$ power conversion efficiency, fabricated by controlled crystallization in the spin-coating process. Adv. Mater. 26, 8179-8183 (2014).

23. M. Xiao, F. Huang, W. Huang, Y. Dkhissi, Y. Zhu, J. Etheridge, A. Gray-Weale, U. Bach, Y.B. Cheng, L. Spiccia, A fast deposition-crystallization procedure for highly efficient lead iodide perovskite thin-film solar cells. Angew. Chem. Int. Ed. 53, 9898-9903 (2014).

24. N. J. Jeon, J. H. Noh, Y. C. Kim, W. S. Yang, S. Ryu, S. I. Seok, Solvent engineering for high-performance inorganic-organic hybrid perovskite solar cells. Nat. Mater. 13, 897-903 (2014).

25. E. Dulkeith, M. Ringler, T. A. Klar, J. Feldmann, Gold nanoparticles quench fluorescence by phase induced radiative rate suppression. Nano Lett. 5, 585-589 (2005).

26. S. Gonzalez-Carrero, R. E. Galian, J. Pérez-Prieto, Maximizing the emissive properties of $\mathrm{CH}_{3} \mathrm{NH}_{3} \mathrm{PbBr}_{3}$ perovskite nanoparticles. J. Mater. Chem. A 3, 9187-9193 (2015).

27. R. Lindblad, N. K. Jena, B. Philippe, J. Oscarsson, D. Bi, A. Lindblad, S. Mandal, B. Pal, D. D. Sarma, O. Karis, H. Siegbahn, E. M. J. Johansson, M. Odelius, H. Rensmo, Electronic structure of $\mathrm{CH}_{3} \mathrm{NH}_{3} \mathrm{PbX}_{3}$ Perovskite: dependence on the halide moiety. J. Phys. Chem. C 119, 1818-1825 (2015).

28. I. A. Shkrob, T. W. Marin, Charge trapping in photovoltaically active perovskites and related halogenoplumbate compounds. J. Phys. Chem. Lett. 5, 1066-1071 (2014).

29. P. Schulz, E. Edri, S. Kirmayer, G. Hodes, D. Cahen, A. Kahn, Interface energetics in organometal halide perovskite-based photovoltaic cells. Energy Environ. Sci. 7, 1377-1381 (2014).

30. J. Kanbe, H. Onuki, R. Onaka, Photoelectron spectra of $\mathrm{PbCl}_{2}, \mathrm{PbBr}_{2}$ and similar materials. J. Phys. Soc. Jpn. 43, 1280-1285 (1977).

31. Q. Chen, H. Zhou, T.-B. Song, S. Luo, Z. Hong, H.-S. Duan, L. Dou, Y. Liu, Y. Yang, 
Controllable self-induced passivation of hybrid lead iodide perovskite toward high performance solar cells. Nano Lett. 14, 4158-4163 (2014).

32. D. Shi, V. Adinolfi, R. Comin, M. Yuan, E. Alarousu, A. Buin, Y. Chen, S. Hoogland, A. Rothenberger, K. Katsiev, Y. Losovyj, X. Zhang, P. A. Dowben, O. F. Mohammed, E. H. Sargent, O. M. Bakr, Low trap-state density and long carrier diffusion in organolead trihalide perovskite single crystals. Science 347, 519-522 (2015).

33. S. D. Stranks, G. E. Eperon, G. Grancini, C. Menelaou, M. J. P. Alcocer, T. Leijtens, L. M. Herz, A. Petrozza, H. J. Snaith, Electron-hole diffusion lengths exceeding 1 micrometer in an organometal trihalide perovskite absorber. Science 342, 341-344 (2013).

34. R. Sheng, A. Ho-Baillie, S. Huang, S. Chen, X. Wen, X. Hao, M. A. Green, Methylammonium lead bromide perovskite-based solar cells by vapor-assisted deposition. $J$. Phys. Chem. C, 119, 3545-3549 (2015).

35. J. C. De Mello, H. F. Wittmann, R. H. Friend, An improved experimental determination of external photoluminescence quantum efficiency. Adv. Mater. 9, 230-232 (1997).

36. X. Qin, H. Dong, W. Hu, Green light-emitting diode from bromine based organic-inorganic halide perovskite, Sci. China Mater. 58, 186-191 (2015).

37. J. I. Langford, A. J. C. Wilson, Scherrer after sixty years: a survey and some new results in the determination of crystallite size. J. Appl. Cryst. 11, 102-113 (1978).

38. J. F. Moulder, W. F. Stickle, P. E. Sobol, K. D. Bomben, Handbook of X-ray photoelectron spectroscopy Ch. II (Physical Electronics, Inc., Minnesota, United States of America, 1992).

39. A. F. van Driel, I. S. Nikolaev, P. Vergeer, P. Lodahl, D. Vanmaekelbergh, W. L. Vos, Statistical analysis of time-resolved emission from ensembles of semiconductor quantum dots: Interpretation of exponential decay models. Phys. Rev. B 75, 035329 (2007).

40. L. Gil-Escrig, G. Longo, A. Pertegás, C. Roldán-Carmona, A. Soriano, M. Sessolo, H. J. Bolink, Efficient photovoltaic and electroluminescent perovskite devices. Chem. Commun. 51, 569-571 (2015).

41. P. E. Shaw, A. Ruseckas, I. D. W. Samuel, Exciton diffusion measurements in poly(3hexylthiophene). Adv. Mater. 20, 3516-3520 (2008).

42. P. E. Shaw, Measurements of exciton diffusion in conjugated polymers. PhD Thesis, University of St Andrews (2009).

43. A. E. McKinnon, A. G. Szabo, D. R. Miller, The deconvolution of photoluminescence data. J. Phys. Chem. 81, 1564-1570 (1977).

Acknowledgements: This work was partially supported by Samsung Research Funding Center of Samsung Electronics under Project Number SRFC-MA-1402-07. Aditya Sadhanala has been supported by the Engineering and Physical Sciences Research Council (UK). All data are available in the main text and the supplementary materials. 


\section{Supplementary Materials:}

Materials and Methods

Figs. S1 to S13

Table S1 to S5

References (35-43) 

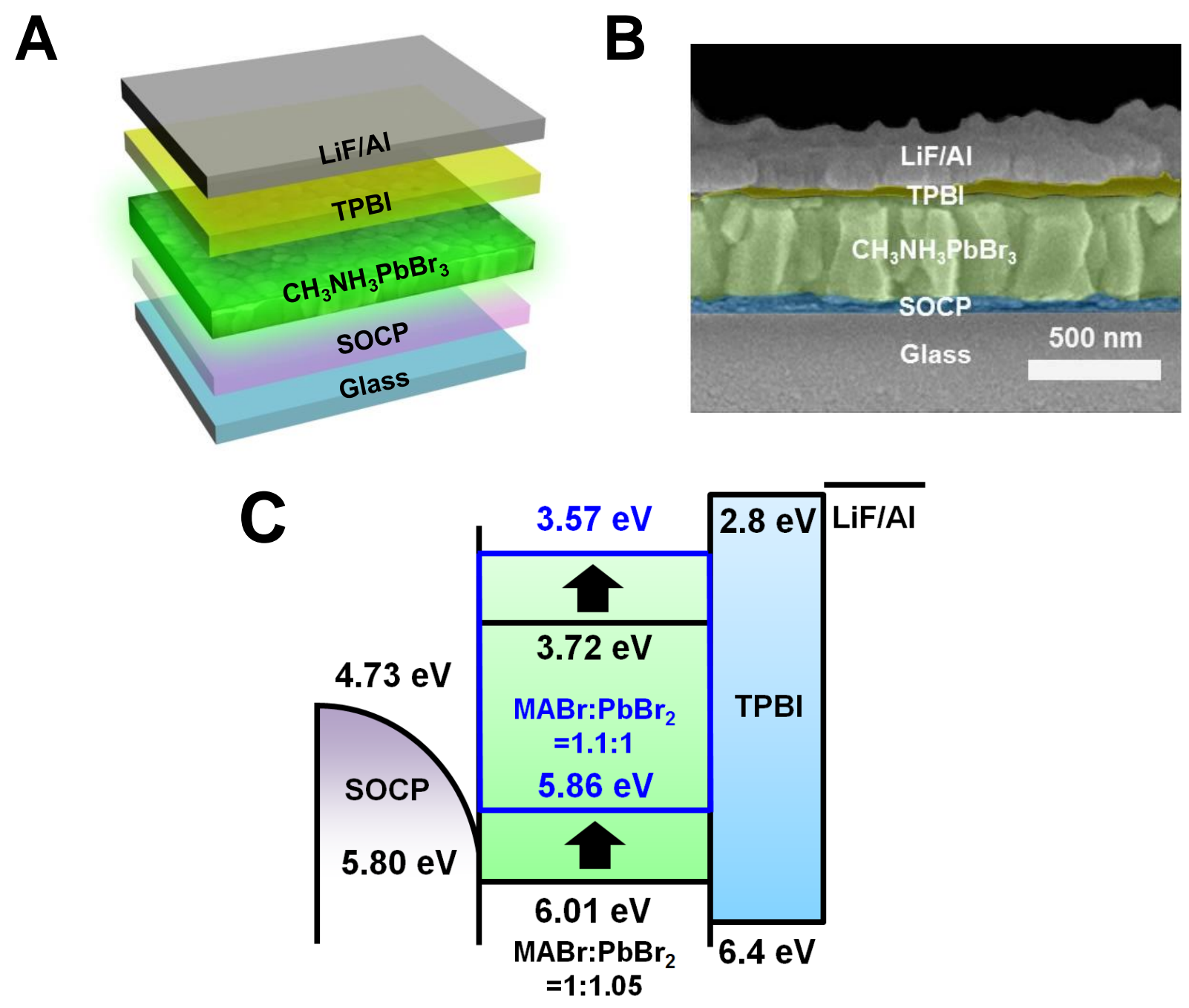

Fig. 1. Schematic illustrations of device structure and its cross-sectional SEM image, and energy band structure. (A) The device structure; perovskite films were characterized without the TPBI layer and LiF/Al electrode. (B) Cross-sectional SEM image of PeLEDs. (C) Energy band diagram of PeLEDs showing decrease of IE with increasing MABr molar proportion 

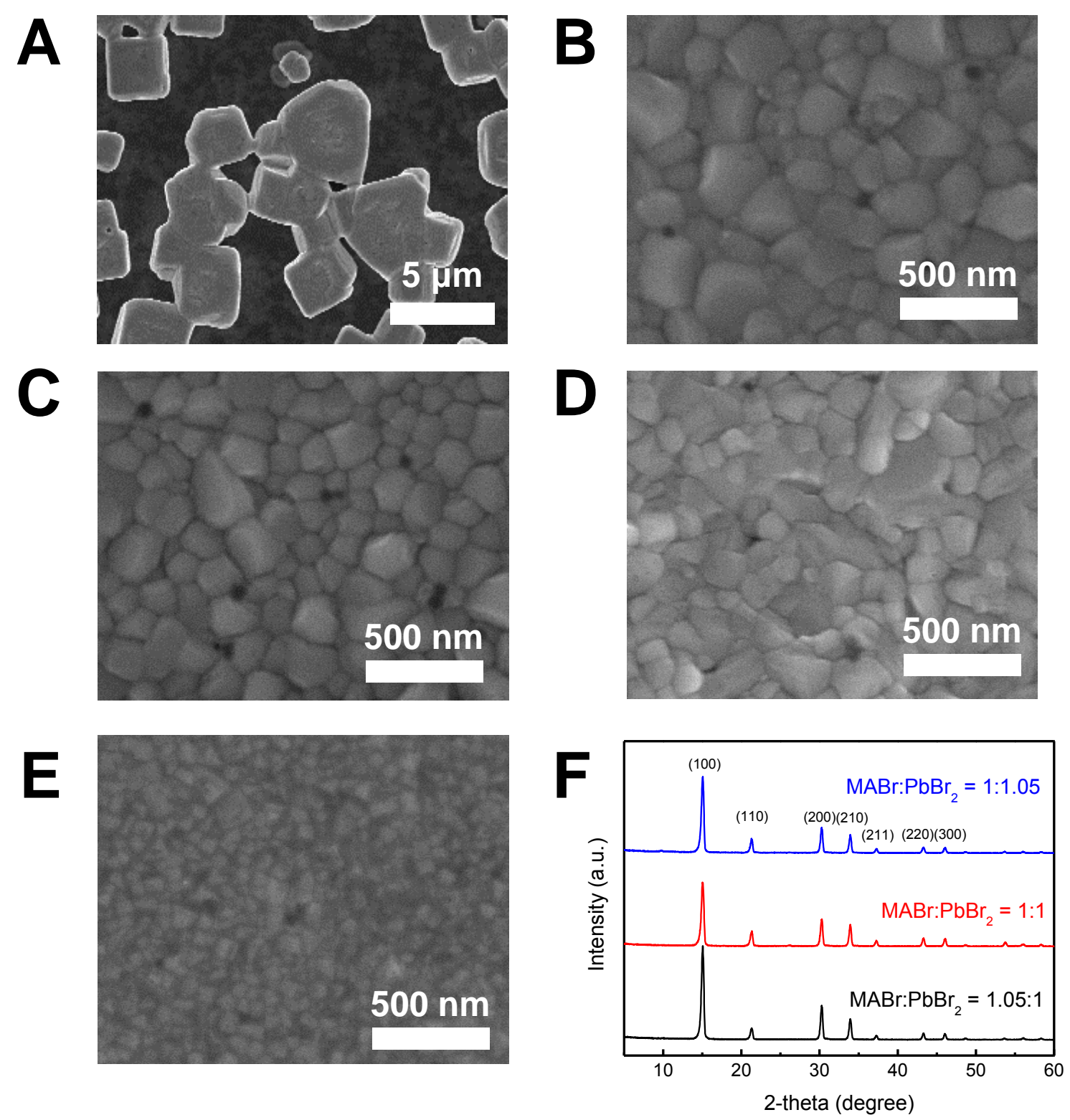

Fig. 2. SEM images and $\mathrm{XRD}$ patterns of $\mathrm{MAPbBr}_{3}$ layers. $\mathrm{SEM}$ images of $\mathrm{MAPbBr}$ layers of (A) $\mathrm{MABr}: \mathrm{PbBr}_{2}=1: 1$ without NCP, (B) 1:1.05, (C) 1:1, (D) 1.05:1 with S-NCP, (E) 1.05:1 with A-NCP. (F) XRD patterns of $\mathrm{MAPbBr}_{3}$ nanograin layers with $\mathrm{MABr}: \mathrm{PbBr}_{2}=1: 1.05,1: 1$ and 1.05:1. 

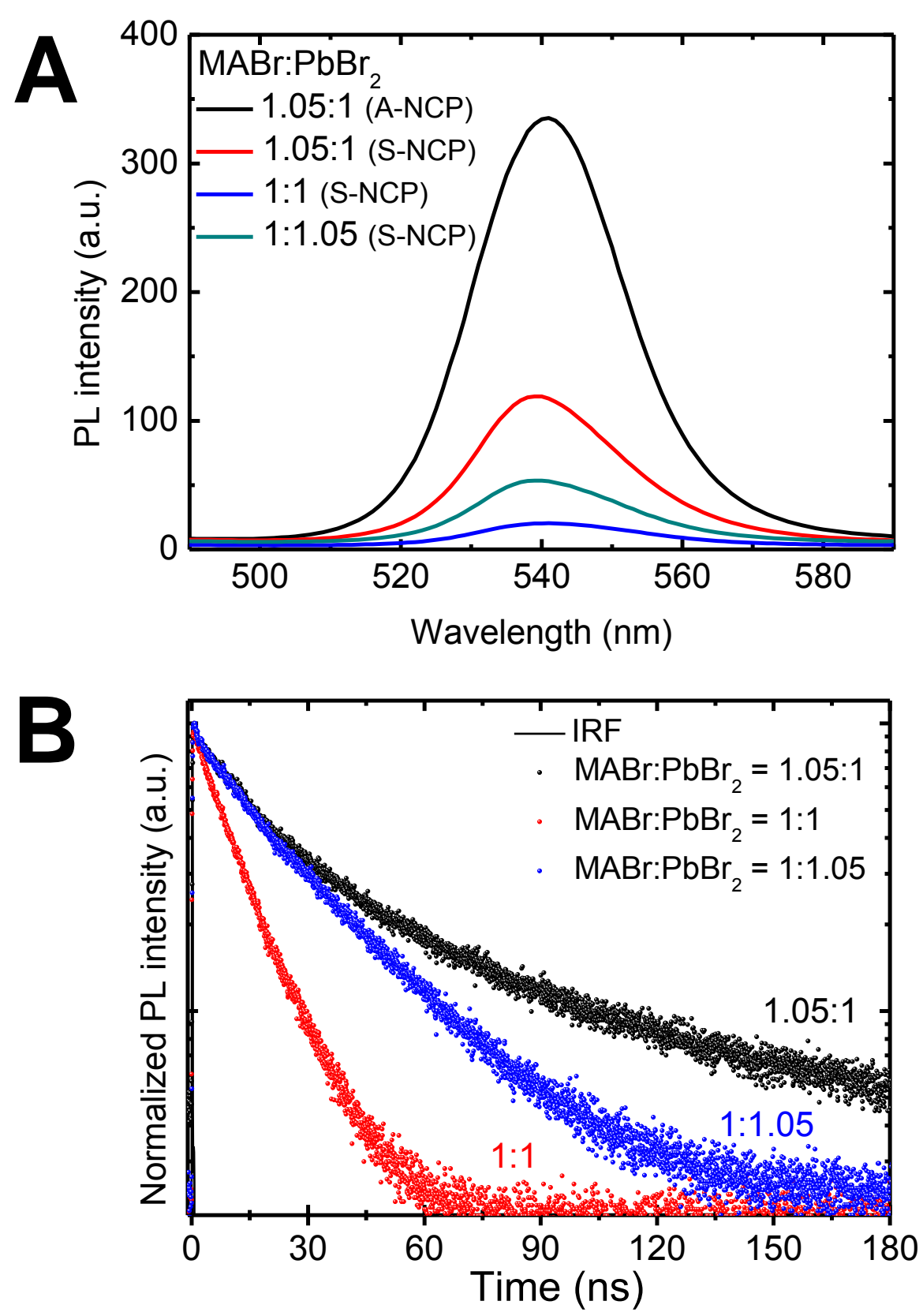

Fig. 3. Steady-state PL spectra and lifetime. (A) Steady-state PL spectra of $\mathrm{MAPbBr}_{3}$ nanograin layers with NCP type and varying molar ratio $\mathrm{MABr}_{\mathrm{PbBr}}$. (B) PL lifetime curves of $\mathrm{MAPbBr}_{3}$ nanograin layers with varying molar ratio $\mathrm{MABr}: \mathrm{PbBr}_{2}$. Black line: instrument response function (IRF). 

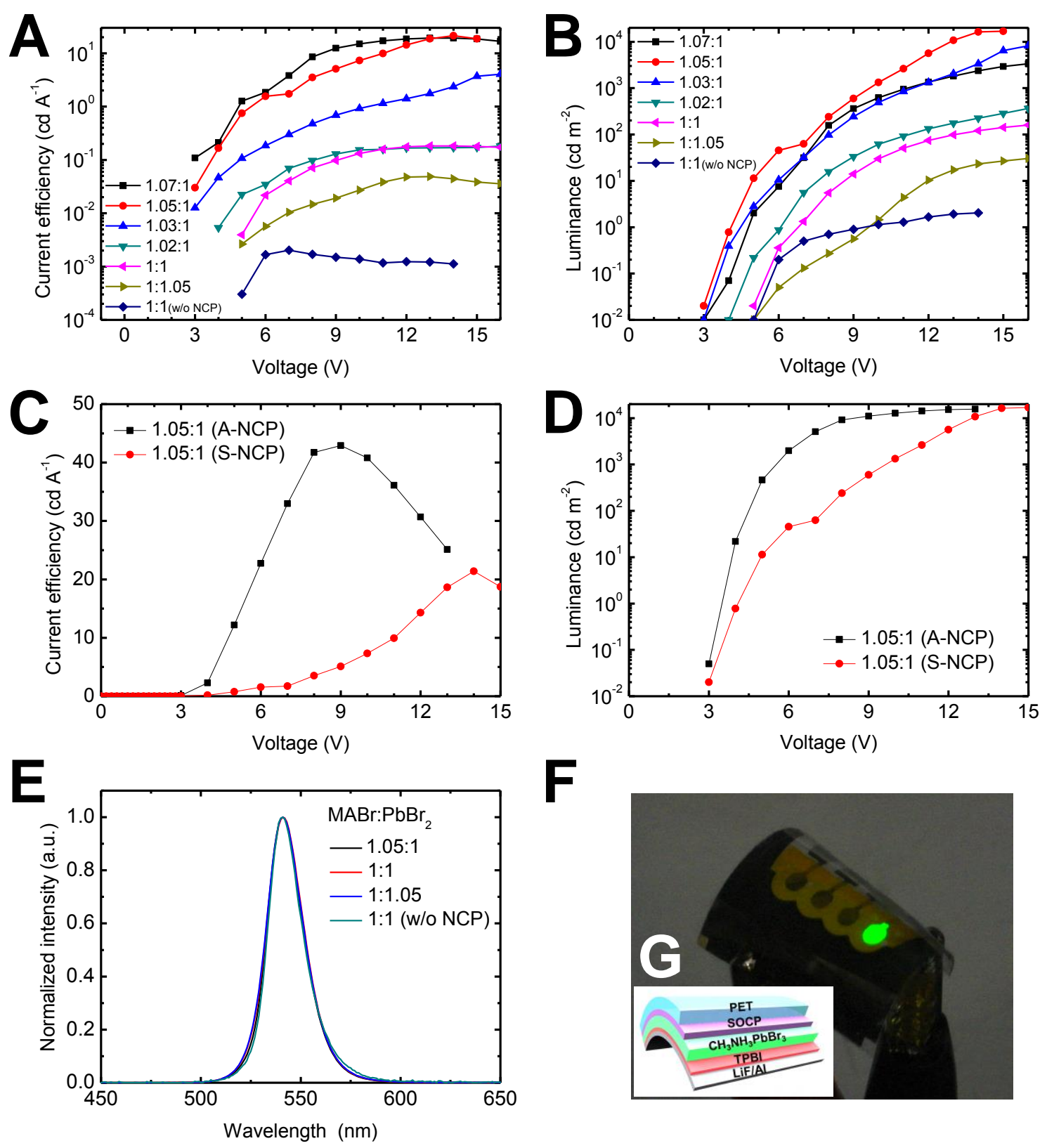

Fig. 4. PeLED characteristics, EL spectra, photograph of PeLED. (A and B) CE and luminance of PeLEDs based on S-NCP and $\mathrm{MAPbBr}_{3}$ nanograin emission layers with varying molar ratio $\mathrm{MABr}_{\mathrm{PbBr}}(-\mathbf{\square}-1.07: 1,-\mathbf{-}-1.05: 1,-\mathbf{\Delta}-1.03: 1,-\boldsymbol{\nabla}-1.02: 1,-4-$ $1: 1, \longrightarrow-1: 1.05,-\longrightarrow 1: 1$ without NCP). (C and D) CE and luminance of PeLEDs based on A-NCP and $\mathrm{MAPbBr}_{3}$ nanograin emission layers. (E) EL spectra of PeLEDs. (F) Photograph of a flexible PeLED, and (G) its device structure. 
Table 1. Maximum CE of PeLEDs depending on NCP and the molar ratio MABr:PbBr 2 .

\begin{tabular}{c|cc}
\hline MABr:PbBr ${ }_{2}$ & NCP type & Max. CE (cd A $\mathbf{~}^{\mathbf{1}}$ ) \\
\hline $1.05: 1$ & A-NCP & 42.9 \\
\hline $1.07: 1$ & & 19.3 \\
$1.05: 1$ & & 21.4 \\
$1.03: 1$ & & 4.03 \\
$1.02: 1$ & S-NCP & 0.457 \\
$1: 1$ & & 0.183 \\
$1: 1.05$ & & $4.87 \times 10^{-2}$ \\
\hline $1: 1$ & Without NCP & $2.03 \times 10^{-3}$ \\
\hline
\end{tabular}

